\section{Idiotype vaccines in the clinic}

To the editor-In an excellent News \& Views article in the June issue ${ }^{1}$, Constantin Bona comments on two papers $^{2,3}$ reporting promising results of idiotype vaccine experiments in mice. Bona suggests that anti-idiotype antibodies have not been adopted as cancer vaccines in humans and speculates that three critical issues may limit the use of antiidiotype antibodies in humans. In fact, we have patient data showing that these issues are not a problem.

First, Bona speculates that murine antibodies by virtue of their xenogeneic origin elicit a strong antibody response in humans, which neutralizes the murine antibodies, thus diminishing their halflife through rapid clearance. Although this is true for 'passive' intravenously administered murine Ab1 antibodies, it is not the case for 'active' immunization with murine anti-idiotype $\mathrm{Ab} 2$ antibody vaccines. It is most likely that the human anti-mouse antibodies bind to the antiidiotype antibodies and the entire complex is endocytosed by antigen presenting cells. We have convincing data on more than 200 patients treated with four different murine anti-idiotypic antibodies that a profound humoral and cellular response is elicited ${ }^{4-7}$. We see high titer $\mathrm{Ab} 3\left(\mathrm{Ab} 1^{\prime}\right)$ responses as well as CD4 T-cell proliferative responses in cancer patients after repeated vaccinations. The human anti-mouse antibody responses have never been a problem in our lab.

The second concern was with the duration of the immune response. Although it is true that the immunity from a single vaccination is not necessarily long-lasting, we have boosted patients monthly for more than four years. They have continued to generate an immune response with $\mathrm{Ab3}$ titers ranging from 40 to $120 \mathrm{mcg} / \mathrm{ml}$. The vaccine is well tolerated, with only swelling and erythema at the site of injection and minimal systemic symptoms. The monthly schedule has been acceptable to all of our patients and we have not had a single patient taken off study for toxicity.

The final issue was that anti-idiotype antibodies primarily generate an IgM immune response. This is not the case with our vaccines. The predominant immunoglobulin response is IgG, distributed among all of the subclasses, but mostly IgG1. In addition, patient's Ab3 sera routinely mediate antibody-dependent cellular cytotoxicity. Most of our patients have also demonstrated idiotypic-specific and antigen-specific CD4 helper T-cell responses. Like the antibody responses, the T-cell responses are long lasting and continue to be maintained over the course of vaccine therapy.

\title{
No immunity for the elderly
}

To the editor-Nature Medicine's recent supplement on vaccines (Volume 4, pages 472-534 May 1998) is timely and certain to stimulate public and research interest in vaccinology. However, the problem of vaccination of the elderly was not addressed. Although the impact of childhood vaccination is generally accepted and well understood, public awareness of the
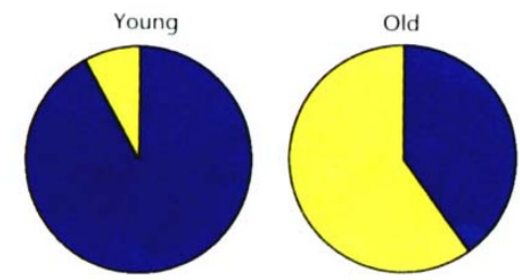

Percentage of persons with ( $\square$ ) and without $(\square)$ protective antibody titers to tetanus. Young ( $<30$ years, $n=30$ ) and old ( $>65$ years, $n=32$ ) healthy volunteers selected according to criteria of the SENIEUR protocol for immunogerontological studies of the European Community's Concerted Action Program on Aging' were analyzed. advantages of immunization in the elderly is not. This is unfortunate given the demographic change to an elderly population taking place in many countries. Infectious diseases remain a major cause of morbidity and mortality in the elderly and much of this could be prevented by appropriate vaccination. It is disturbing, for example, that many elderly people do not have immunity even against tetanus, against which vaccines have been available for decades ${ }^{1,2}$ (Fig.). Similar data exist for influenza (even in those who had been vaccinated ${ }^{3}$ ) and pathogens such as pneumococci, mycobacterium tuberculosis and corynebacterium diphtheriae. This low protection rate against preventable diseases is partly due to lack of information and low vaccination acceptance among the elderly and partly due to the gradual age-related decline in the functional capacity of the immune system ${ }^{4}$.

Efforts should be made to draw public attention to the problem of vaccination in
Our data indicate that the anti-idiotype vaccine approach is ready for clinical use. We see patients with long-lasting IgG humoral and cellular immune responses to a variety of tumor-associated antigens including carcinoembryonic antigen, human milk fat globule antigen, the GD2 disialoganglioside and a highly restricted T-cell antigen.

Kenneth A. Foon \& Malaya

BHATTACHARYA-CHATTERJEE

Markey Cancer Center

University of Kentucky

800 Rose Street, Room 140

Lexington, Kentucky 40536-0093 USA

1. Bona, C.A. Idiotype vaccines: Forgotten but not gone. Nature Med. 4, 668-669 (1998).

2. Magliani, W. et al. Neonatal mouse immunity against group B streptococcal infection by maternal vaccination with recombinant antiidiotypes. Nature Med. 4, 705-709 (1998).

3. Ruiz, P.J. et al, Immunity to mutant p53 and tumor rejection induced by idiotypic immunization. Nature Med. 4, 710-712 (1998).

4. Foon, K.A. et al. Active immunity to the carcinoembryonic antigen in patients treated with an anti-idiotype antibody vaccine. / Clin. Invest. 96, 334-342 (1995).

5. Foon, K.A. et al. Immune responses in patients with cutaneous T-cell lymphoma treated with an anti-idiotype antibody mimicking a highly restricted T-cell antigen. Clin. Cancer Res 1,1285-1294 (1995).

6. Foon, K.A. et al. Clinical and immune responses in advanced colorectal cancer patients treated with anti-idiotype monoclonal antibody vaccine that mimics the carcinoembryonic antigen. Clin. Cancer Res. 3,1267-1276 (1997).

7. Foon, K.A. et al. Antibody responses in melanoma patients immunized with an anti-idiotype antibody mimicking disialoganglioside GD2. Clin. Cancer Res. 4,1117-1124 (1998).

the elderly. The design of age-specific vaccination strategies and the development of vaccines that are suitable to overcome the defects of the aging immune system should be a major goal of the vaccine community.

1. Gergen, P.j. et al. A population-based serologic survey of immunity to tetanus in the United States. New Engl. J. Med. 32, 761-766 (1995).

2. Steger, M.M. et al. Vaccination against tetanus in the elderly: Do recommended vaccination strategies give sufficient protection? Lancet 348,762 (1996).

3. Beyer, W.E.P. et al. Antibody induction by influenza vaccine in the elderly. Vaccine 7, 385-394 (1989)

4. Wick, G. \& Grubeck-Loebenstein, B. Primary and secondary alterations of immune reactivity in the elderly: Impact of dietary factors and disease. immunol. Rev. 160, 171-184 (1997).

5. Lighthart, G.J. et al. Admission criteria for immunogerontological studies in man: The Senieur protocol. Mech. Ageing Dev. 28, 47-55 (1984).

Beatrix Grubeck-Loebenstein, Peter Berger, Maria SaURWEIN-TeissL, KARINA ZisTERER \& GEORG WICK Institute for Biomedical Aging Research of the Austrian Academy of Sciences

Rennweg 10, A-6020 Innsbruck, Austria

email: Beatrix.Grubeck@oeaw.ac.at 